\title{
Mathematical and Computer Simulation of Visual Acuity in Imaging Devices
}

\author{
Alla V. Shymanska
}

\begin{abstract}
Image converters and intensifiers give access to a larger spectral domain of electromagnetic radiation than the human eye. They amplify low light images and increase the eye sensitivity. The visual acuity, and therefore, the range of vision of any image detector, is limited by optical properties of the detector, such as a modulation-transfer-function (MTF) and the value of noise, produced by the device. This paper presents the methods and computational algorithms for calculating MTF and the noise factor of image converters and intensifiers which incorporate an inverting electron optical system and a microchannel plate as an amplifier. The approach, presented here, enables one to improve the visual acuity of the device.
\end{abstract}

Index Terms-Image device, modulation-transfer-function, noise factor, visual acuity.

\section{INTRODUCTION}

Although the human eye is a good and adaptable photon detector, it has some physical limitations. The limitations of visual perception include limitations of the eye as radiation detector: wavelength and sensitivity limitations, and limitations of the eye as an image detector what includes resolving power and contrast perceptibility [1].

These limitations can be improved by using image converters and intensifiers where the original image, created from wave lengths outside the visible region, is converted into the visible image. Image converters and intensifiers give access to a larger spectral domain of electromagnetic radiation than the human eye, amplify low light images and increase the eye sensitivity.

Distinction of small details of an object, and therefore, the range of vision of any image detector, are limited by optical properties of the detector. Such properties are usually characterized by the modulation-transfer-function (MTF) and by the value of noise, produced by the device. The MTF describes the resolution over the image area of an image device [2]-[4], and gives the dependence of the output contrast on the spatial frequency. The noise factor $\mathrm{F}$, which is a measure of the loss of available information, can be written as [5]:

$$
F=\frac{(S / N)_{\text {in }}^{2}}{(S / N)_{\text {out }}^{2}},
$$

where $(S / N)_{\text {in }}$ and $(S / N)_{\text {out }}$ are ratios of the input signal (S) to the noise $(\mathrm{N})$ and the output signal to the noise

Manuscript received March 8, 2014; revised May 6, 2014.

Alla V. Shymanska is with the Auckland University of Technology, Auckland 1142, New Zealand (e-mail: alla.shymanska@aut.ac.nz). respectively.

The noise factor and MTF of the device affect a visual acuity which defines the size of the smallest visible detail as a function of brightness for different values of contrast. The angular resolution (the visual acuity) for different levels of illumination can be given by the formula [1]:

$$
L \alpha^{2}=\frac{7.5 \times 10^{10}(S / N)_{\min }^{2}}{D^{2} \tau \theta P C^{2}}
$$

where $L, k d / m^{2}$, is the illumination; $\alpha$, min, is the angle of vision of an object detail (visual acuity); $\mathrm{C}$ is a contrast of an image; $(S / N)_{\min }$ is the minimal ratio of the signal to noise, which required to detect details of an object.

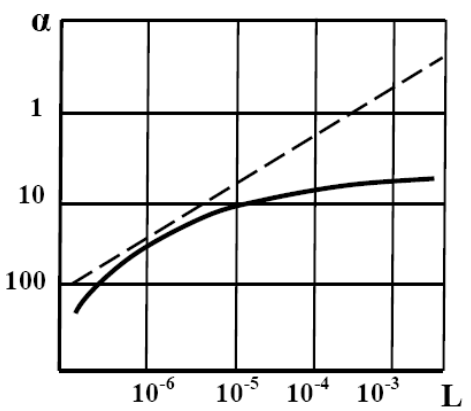

Fig. 1. Dependence of the visual acuity ( $\alpha \min$ ) on the illumination

$$
\left(L \mathrm{kd} / \mathrm{m}^{2}\right) \text {. }
$$

Fig. 1 shows the typical curve of the visual acuity of an image detector [1]. For the high illumination, MTF reduces the contrast to the level, lower than the contrast of the observable object, and deteriorates the visual acuity. The image device is the source of the extra noise, and the ratio $(S / N)_{\text {out }}$ decreases as $(S / N)_{\text {out }}=(S / N)_{\text {in }} \sqrt{F}$. Respectively, the ratio $(S / N)_{\text {in }}$, needed to detect an object, increases in $\sqrt{F}$ times. This leads to deterioration of the visual acuity especially in case of low illumination. Therefore, improvement of visual acuity of image detectors relates to improvement of the detector's MTF, and to reduction of its noise factor. It is especially important for the night vision devices.

This paper presents methods and computational algorithms for calculating MTF and the noise factor of image converters and intensifiers which incorporate an inverting electron optical system (EOS) and a microchannel plate (MCP) as an amplifier.

In such case, the process of image conversion and intensification is: (a) electromagnetic radiation from a certain part of the spectrum is absorbed by a photocathode; (b) an optical image is converted into an electron image by means of 
photoemission; (c) the photoelectrons are accelerated and focused by EOS, and intensified by the MCP; (d) the intensified electron image is converted into a visible image by a luminescent process on a screen. Fig. 2 shows a cross section of such device.

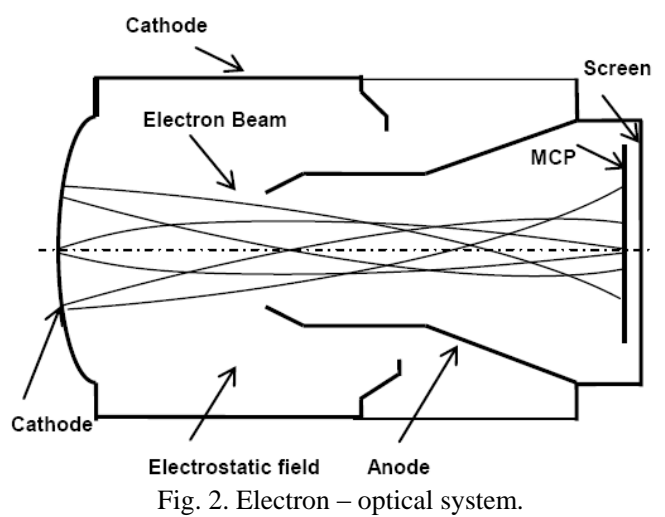

The MCP is a thin plate made up of a large number of single parallel channels, each of which acts as an electron multiplier [2], [4], [6]. For each primary electron entering a channel, a large pulse of electrons will exit as a result of multiple collisions of both primary and secondary electrons with the inner secondary-emission layer. It is a compact, efficient amplifier of two-dimensional electron-images and provides several advantages such as very high gain, good resolution and small size. Poor noise characteristics are the main drawback of systems with channel amplification.

A computational method for simulation of stochastic processes of an electron multiplication in microchannel electron amplifiers, developed by the author [4], is used for investigation of the noise factor. The method is based on 3D Monte Carlo (MC) simulations and theorems about serial and parallel amplification stages proposed by the author. The method is used to show how the input ratio of the signal to the noise is transforming to the output one, and how different multiplication stages contribute to the noise factor of the system.

The analysis of MTF of the image device includes calculations of the MTF of the EOS and MTF of the channel multiplier. Such calculations are based on numerical calculation of the field distribution inside the device and the channel, trajectories of the photo-and secondary electrons [7].

\section{Computational Analysis of MTF}

\section{A. Motion of Electrons in the Potential Field}

Calculation of the electrostatic field in the device with rotational symmetry (Fig. 2) is a matter of finding a solution to the Laplace's partial differential equation expressed in cylindrical coordinates as follows:

$$
\frac{\partial^{2} U}{\partial z^{2}}+\frac{1}{r} \frac{\partial U}{\partial r}+\frac{\partial^{2} U}{\partial r^{2}}=0,
$$

where $U(r, z)$ is the potential at any point $(z, r), z$ is the coordinate along the axis of symmetry and $r$ is the off-axis radius.
It is the classical mixed problem for the equation of Laplace in some region with Dirichlet and Neumann boundary conditions. The finite difference method is used to obtain the field distribution [7].

Fig. 3 shows the computational results of the potential distribution (given by the equipotential lines) in the cross-section of the EOS.

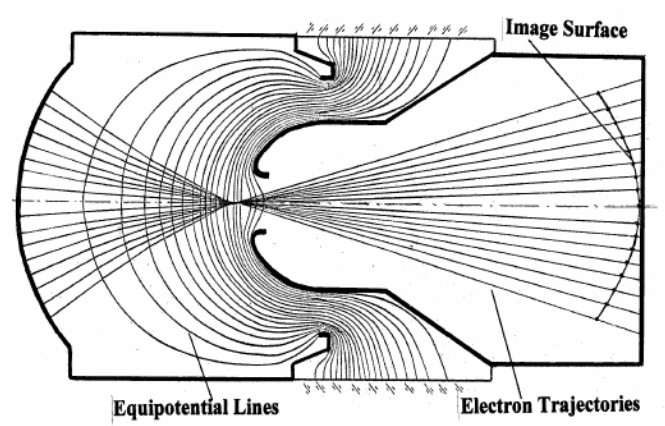

Fig. 3. Equipotential lines and central meridional trajectories of electron beams.

An electrostatic lens, formed by MCP-screen field penetration into channels, at the output of a channel has a significant influence on the electron trajectories and the spatial resolution [7]. The potential distribution in the field of the lens depends on the field intensity in the MCP-screen gap, the channel diameter, and the sputtering depth of the contact layer at the channel output. Fig. 4 shows the computational results of the electrostatic lens at the exit of a single channel.

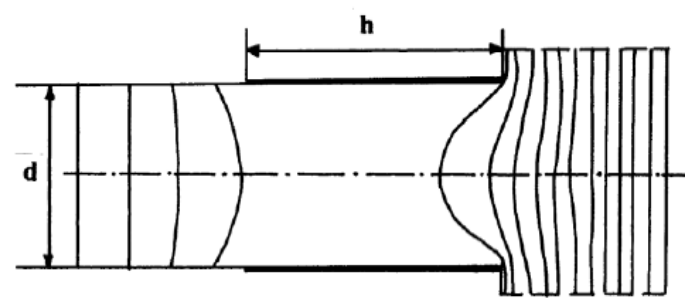

Fig. 4. Electrostatic field at the exit of the channel.

In the cylindrical coordinate system, equations of motion of electrons can be written as:

$$
\begin{aligned}
& \frac{d^{2} z}{d t^{2}}=\frac{e}{m} E_{z} \\
& \frac{d^{2} r}{d t^{2}}=\frac{e}{m} E_{r}+\frac{r_{0}^{2} V_{\varphi 0}^{2}}{r^{3}} \\
& \frac{d \varphi}{d t}=\frac{r_{0}}{r^{2}} V_{\varphi 0}
\end{aligned}
$$

where $t$ is time, $E_{r}=\frac{\partial U}{\partial r}$ and $E_{z}=\frac{\partial U}{\partial z}$ are the radial and axial components of the strength of the electrostatic field respectively, $r_{0}$ is the initial electron coordinate, $V_{\varphi 0}$ is the initial azimuthal component of the electron velocity, $e$ and $m$ are electron charge and mass respectively.

The system of (4) is solved by the Runge-Kutta method for the electron motion inside the EOS of the device, and in the area of the inhomogeneous electrostatic field of the channel multiplier. The strengths of the electrostatic field at the exit of a single channel and inside the EOS are calculated using 
different interpolating polynomials [7].

Fig. 3 shows central meridional trajectories of the electron beams emitted from the photocathode.

\section{B. Modulation Transfer Function of the Image System}

The MTF of the overall system, at a given spatial frequency, is the product of the MTFs of the elements [2], [3], [7]. Consequently, to evaluate the total MTF of the imaging system with a micro-channel plate, the MTFs of EOS and MCP-screen system should be determined.

The MTF in the image plane for the point on the axis of the symmetry of EOS is calculated using the well known formula:

$$
T=\frac{\int_{0}^{\infty} A(r) J_{0}(2 \pi v r) r d r}{\int_{0}^{\infty} A(r) r d r}
$$

where $J_{0}$ is Bessel's function, $v$ is a spatial frequency, and $A(r)$ is an electron density distribution in the cross section of the axial electron beam on the image surface.

To find the electron density function $A(r)$ let assume that $d N(\varepsilon, \theta, j)$ electrons are emitted from the axial point of the photocathode in a time unit with initial energies in the interval $[\varepsilon, \varepsilon+d \varepsilon]$, initial angles $[\theta, \theta+d \theta]$ and $[\varphi, \varphi+d \varphi]$, where $\theta$ and $\varphi$ are meridional and asimuthal angles respectively.

The number of electrons $d N(\varepsilon, \theta, \varphi)$ depends on the total number of the electrons $N_{0}$ emitted from one point of the photocathode and the probability of the emission $P(\varepsilon, \theta, \varphi)$. Therefore,

$$
d N(\varepsilon, \theta, \varphi)=N_{0} d P(\varepsilon, \theta, \varphi)=N_{0} p(\varepsilon, \theta, \varphi) d \varepsilon d \Omega,
$$

where $p(\varepsilon, \theta, \varphi)$ is the probability density, and $d \Omega=\sin \theta d \theta$.

Since $\varepsilon, \theta$ and $\varphi$ are independent random variables then $p(\varepsilon, \theta, \varphi)=p(\varepsilon) p(\theta) p(\varphi)$, where $p(\varepsilon), p(\theta)$, and $p(\varphi)$ are probability density functions of the electron's energy, meridional and azimuthal angles respectively.

Assuming that $p(\varphi)=2 \pi$ is the uniform distribution, the expression for the number of the emitted electrons $d N(\varepsilon, \theta, \varphi)$ can be written as:

$$
\begin{aligned}
& d N(\varepsilon, \theta, \varphi)=N_{0} p(\varepsilon) d \varepsilon p(\theta) \sin \theta d \theta \int_{0}^{2 \pi} p(\varphi) d \varphi= \\
& =N_{0} p(\varepsilon) p(\theta) \sin \theta d \theta d \varepsilon
\end{aligned}
$$

The photoelectrons with the initial energy $\varepsilon$ would have a distance $r$ from the axis of the symmetry at the image surface if their initial angle $\theta$ satisfies a condition $r-f(\varepsilon, \theta)=0$. Using the $\delta$-function the expression for the electron density at the distance $r$ from the axis of the symmetry can be written as:

$$
\begin{aligned}
& d n(r)=\frac{d N(\varepsilon, r)}{2 \pi r}= \\
& =\frac{N_{0} p(\varepsilon)}{2 \pi r} \int_{0}^{\pi / 2} p(\theta) \sin \theta \delta[r-f(\varepsilon, \theta)] d \theta d \varepsilon
\end{aligned}
$$

Integrating (6) with respect to energy $\varepsilon$ the electron density function $A(r)$ can be expressed as

$$
\begin{aligned}
& A(r)= \\
& =\int_{0}^{\varepsilon_{0}} \frac{N_{0} p(\varepsilon)}{2 \pi r} \int_{0}^{\pi / 2} p(\theta) \sin \theta \delta[r-f(\varepsilon, \theta)] d \theta d \varepsilon
\end{aligned}
$$

Substituting (7) to (5) the expression for the modulation transfer functions can be written as:

$$
\begin{aligned}
& T(v)= \\
& =\frac{\int_{0}^{\infty} \int_{0}^{\varepsilon 0} p(\varepsilon)\left\{\int_{0}^{\pi / 2} p(\theta) \sin \theta \delta[r-f(\theta, \varepsilon)] d \theta\right\} d \varepsilon J_{0}(2 \pi v r) d r}{\int_{0}^{\infty} \int_{0}^{\varepsilon_{0}} p(\varepsilon)\left\{\int_{0}^{\pi / 2} p(\theta) \sin \theta \delta[r-f(\theta, \varepsilon)] d \theta\right\} d \varepsilon d r}
\end{aligned}
$$

Using the property of the $\delta$-function that $\int_{0}^{\infty} \varphi(x) \delta\left(x-x_{0}\right) d x=\varphi\left(x_{0}\right)$ the final formula for the modulation transfer functions can be obtained:

$$
T(v)=\frac{\int_{0}^{\varepsilon_{0}} p(\varepsilon) \int_{0}^{\pi / 2} p(\theta) \sin \theta J_{0}[2 \pi v f(\theta, \varepsilon)] d \theta d \varepsilon}{\int_{0}^{\varepsilon_{0}} p(\varepsilon) \int_{0}^{\pi / 2} p(\theta) \sin \theta d \theta d \varepsilon},
$$

where $p(\varepsilon), p(\theta)$ are energy and angular distributions of the electrons respectively (here the angular distribution is described by Lambert's law, $p(\theta)=\cos \theta$, and the electron energy is chosen from the parabolic function $p(\varepsilon)=\frac{6 \varepsilon}{\varepsilon_{0}}\left(1-\frac{\varepsilon}{\varepsilon_{0}}\right) ; \varepsilon_{0}$ is the maximal possible initial energy of the photoelectron; $r=f(\varepsilon, \theta)$ is the distribution of the electron radial coordinates at the image surface which can be written as:

$$
r(z)=2 \sqrt{\frac{\varepsilon}{E_{0}}} \sin \theta u_{1}(z)-\frac{2 \varepsilon}{E_{0}} \sin \theta \cos \theta u_{2}(z),
$$

where $E_{0}$ is the strength of the electrostatic field near the photocathode; $u_{1}(z)$ and $u_{2}(z)$ are two particular solutions of the equation of the motion of paraxial electrons. $u_{1}(z)$ and $u_{2}(z)$ are calculated as described above.

Formula (5) is used in computations of the MTF of the $\mathrm{MCP}$-screen system as well. The electron density distribution $A(r)$ in the image of an individual channel is determined from the arrival coordinates of the electrons at the screen. The multiplication process of the electron flux in the channel was modeled using MC simulations as it is described below.

Taking into account the discreet nature of the determination of $A(r)$, and using the relationship $\int x^{n} J_{n-1}(x) d x=x^{n} J_{n}(x)$, the calculating formula for MTF of the channel multiplier can be written: 


$$
T=\frac{\sum_{i=1}^{n} A_{i}\left(r_{i}\right)\left[r_{i+1} J_{1}\left(2 \pi v r_{i+1}\right)-r_{i} J_{1}\left(2 \pi v r_{i}\right)\right]}{\pi v \sum_{i=1}^{n} A_{i}\left(r_{i}\right)\left(r_{i+1}^{2}-r_{i}^{2}\right)},
$$

Fig. 5 shows calculated results of the dependence of the EOS's MTF on the potential $U_{a}$, applied between the cathode and the anode.

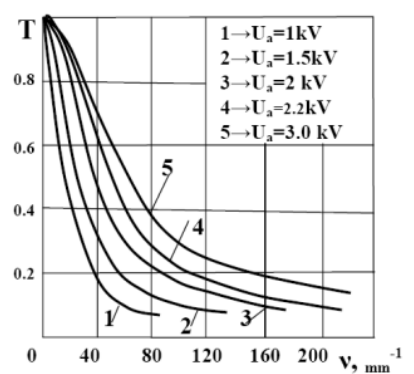

Fig. 5. MTF's of the EOS for the different voltage between the cathode and anode.

Fig. 6 displays the MTFs of the MCP-screen system, where the solid curves represent calculations and dashed curve represents experimental results. (The experimental data here and later was provided by A.M.Tyutikov, State Optic Institute, St Petersburg.) The distance between MCP and the screen $D=1 \mathrm{~mm}$ for the curves (2) and (3), and $D=0.4 \mathrm{~mm}$ for the curve (1).

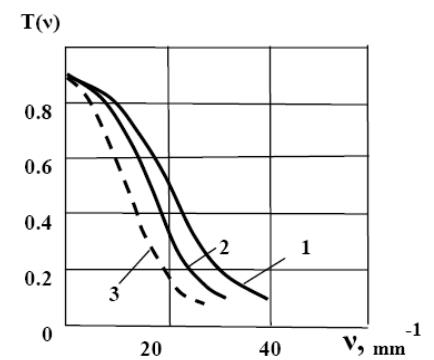

Fig. 6. MTF of the MCP-screen (the solid curves represent calculations and dashed curve represents experiment).

The final MTF of the image system is the product of the MTFs of the EOS (Fig. 5\}) and MCP (Fig. 6). Fig. 7 shows the total MTF of such imaging system where the solid curve represents calculations $(d=10 \mu \mathrm{m})$ and dashed curve represents experiment $(d=12 \mu \mathrm{m})$ for the MCP-screen gap $D=0.4 \mathrm{~mm}$.

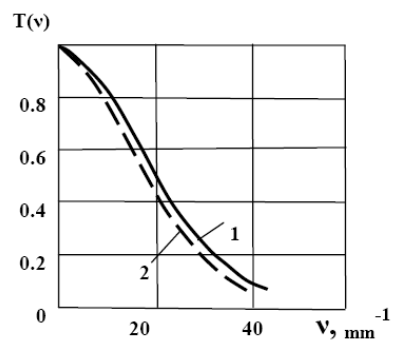

Fig. 7. Final MTF of the imaging system (the solid curve represents calculations and dashed curve represents experiment).

\section{NOISE FACTOR}

\section{A. Simulation of Stochastic Processes}

The following real physical picture was implemented in the model. The electrons of a primary monochromatic parallel beam are incident on the input plane of a microchannel amplifier. Electrons, entering the channel, hit the channel walls at different angles and different incidence coordinates (Fig. 8).

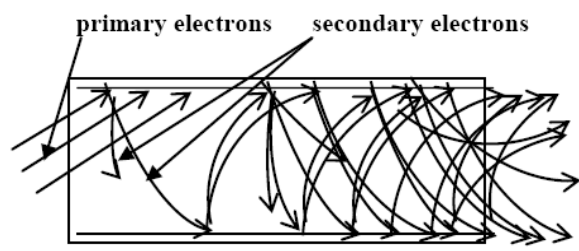

Fig. 8. Electron multiplication in the channel.

After the first collision the primary electrons produce secondary electrons with different emission energy and directions. The secondary emission yield (SEY) of the first collision and the length along which subsequent amplification occurs in the channel are different. The secondary electrons are multiplied until they leave the channel. When all the electrons have emerged from the channel, the yield of the individual pulse is known. The gain of individual pulses is fluctuated considerably, and the pulse amplitude distribution at the output of the channel has a negative exponential form [3]-[5]. The Poisson distribution at the beginning of the channel changes to the negative exponential function. The length of a channel, where the amplitude distribution changes from the peaked one to the negative exponential distribution, is determined in [4] and called 'the effective length of a channel', which is equal half of the channel's length.

The mean gain and the variance of the amplitude distribution at the output of the system define the noise factor of the amplifier which is greater for the negative exponential distribution than for a peaked amplitude distribution.

The entire multiplication process can be split into sequential stages and/or parallel multiplication paths, and how it is done depends on particular investigation. If the input signal amplification is represented as a sequence of transformations (each of which is characterized by the mean and variance), then one can speak of serial amplification stages. The mean and variance of the entire multiplication process can be calculated using the mean and variance of the separate sequential stages.

If $p_{k}(v)$ is the probability distribution of the number of particles at the output of the k-th stage, produced by one particle at its input, $m_{k}$ is the mean and $d_{k}$ is the variance of the $p_{k}(v)$, then, using the generating function of the probability distribution $p_{k}(v)$, we obtain the mean $M$, and variance $D$ of the amplitude distribution $P_{N}(v)$ after the $N$-th stage:

$$
\begin{aligned}
& M=\prod_{k=0}^{N} m_{k} \\
& D=\sum_{k=0}^{N} d_{k} \prod_{i=0}^{k-1} m_{i} \prod_{j=k+1}^{N} m_{j}^{2}
\end{aligned}
$$

The expressions (10) constitute the theorem of serial amplification stages [5].

If the primary particle is multiplied along one of $\mathrm{n}$ possible 
parallel paths, and if each path gives an average of $g_{k}$ particles at the output with a variance of $v_{k}$, then the mean $G$ and the variance $V$ of the amplitude distribution at the output of the system with some parallel amplification paths, can be obtained as:

$$
\begin{gathered}
G=\sum_{k=1}^{n} \rho_{k} g_{k} \\
V=\sum_{k=1}^{n} \rho_{k} v_{k}+\sum_{k=1}^{n} \rho_{k} g_{k}^{2}-G^{2}
\end{gathered}
$$

where $\rho_{k}$ is the probability of choosing the k-th path. Equations (11) constitute the theorem of parallel amplification paths [4].

In the model the multiplication process of a single electron with an initial departure coordinate of $z=0(z$ is the coordinate directed along the channel axis and measured from its beginning) is simulated by $3 \mathrm{D}$ MC methods in a homogeneous field along the effective channel length.

The process of MC simulations uses a random number generating procedure [5] to sample the various distributions such as: the distribution of the actual yield of secondaries after each collision, the emission energy, and the direction of each secondary electron.

The actual number of secondaries generated by the particular collision is a random sample taken from the Poisson distribution

$$
P(v)=\frac{\sigma^{v} e^{-\sigma}}{v !}
$$

where $v$ is the number of secondary electrons produced, $\sigma$ is the Secondary Emission Yield (SEY). The variation of the SEY is defined by a secondary emission function [5]:

$$
\sigma=\sigma_{m}\left[\frac{V}{V_{m}} \sqrt{\cos \theta_{0}}\right]^{\beta} e^{\alpha\left(1-\cos \theta_{0}\right)+\beta\left(1-\frac{V}{V_{m}} \sqrt{\cos \theta_{0}}\right)}
$$

where $V$ is the collision energy in $\mathrm{eV}, \theta_{0}$ is the collision angle (angle between the direction of the primary electron and the normal to the surface), $\sigma_{m}$ is the maximum SEY for the normal incidence $\theta_{0}=0$ ) which is achieved for $V=V_{m}$ (both $V_{m}$ and $\sigma_{m}$ are functions of $\theta_{0}$ ); $\alpha$ and $\beta$ are constants of the channel multiplier surface, and are chosen to fit experimental secondary emission curves at normal incidence.

The energy distribution is described by the formula:

$$
p(\varepsilon)=2.1 \bar{\varepsilon}^{-3 / 2} \sqrt{\varepsilon} \exp (-1.5 \varepsilon / \bar{\varepsilon})
$$

where $\bar{\varepsilon}$ is the mean energy.

Each secondary electron is assigned two emission angles chosen from Lambert's law:

$$
p_{1}(\theta)=\sin 2 \theta \text { and } p_{2}(\varphi)=1 / 2 \pi,
$$

where $\theta$ is the angle between the normal to the surface and emission direction, and $\varphi$ is the azimuthal angle.

The trajectory of each electron is calculated in three dimensions from the ballistic equations, and the position, energy, and angle of the subsequent collisions are determined. The result of each collision is calculated as before and the process is repeated for each secondary electron generated.

The dependence of the mean gain $m(z)$ and variance $d(z)$ on the coordinate $\mathrm{z}$ along the channel axis $0 \leq z \leq L / 2$, where $L$ is the coordinate of the end of the channel) are calculated for a single electron starting out at the beginning of the channel $(z=0)$, using MC simulations. Then, using the theorems of serial and parallel amplification stages, the functions $m(z)$ and $d(z)$ along the entire channel length $(0 \leq z \leq L)$ are calculated.

Modeling the statistical multiplication processes by means of the MC methods for only one electron, emitted at the beginning of the channel, along half of its length reduces considerably the amount of computer time required to investigate the noise characteristics of the channel multiplier

The functions $m(z)$ and $d(z)$ for $0 \leq z \leq L$ and the theorems about serial and parallel amplification stages are used to calculate the mean $M$ and the variance $D$ of the distribution at the output of a channel, and thus determine the noise factor of the channel multiplier.

\section{B. The Noise Factor of the Channel Amplifier}

Assuming that the number of electrons entering the multiplier fluctuates according to Poisson's law [3] with the mean and variance $n_{e}$, we obtain:

$$
(S / N)_{i n}=\left(n_{e} / \sqrt{n_{e}}\right)^{2}=n_{e}
$$

Using the definition of the noise factor (1) and the theorems about serial and parallel amplification stages, expressions for calculating the noise factor can be obtained. The expressions depend on how the entire process is split into a sequence of amplification steps.

The entire amplification process in the channel multiplier can be represented in the form of a sequence of the next several stages.

1) The first observation of electrons, incident at the input of the multiplier (described by the Bernoulli distribution [3]), can be defined as a first stage. If $\gamma$ is the fraction of the front surface of the multiplier exposed to electrons, then the average number of particles entering the channel and the variance can be given by $m_{0}=\gamma$, and $d_{0}=\gamma(1-\gamma)$.

2) The collision of the primary electrons with the wall of the channel is defined as the second stage of the amplification. The distribution of the number of electrons knocked out by one primary electron (with the mean $m_{1}$ and the variance $d_{1}$ ) depends on the properties of the emitter. For a uniform emitter the number of electrons fluctuates according to Poisson's law with $m_{1}=d_{1}=\sigma_{1}$, where $\sigma_{1}$ is the SEY of the emitting surface.

3) Further amplification of the electrons in the channel is regarded as the third stage with the mean gain $m_{2}=m(L)$ and the variance $d_{2}=d(L)$.

Taking into account the contribution of each stage to the 
overall process of amplification and with the help of the formulae (10) and (11) we obtain:

$$
\left(\frac{S}{N}\right)_{\text {out }}^{2}=\frac{M^{2}}{D},
$$

where

$$
M=n_{e} \gamma m_{1} m(L) \begin{aligned}
& D=n_{e}\left[m_{1} m(L)\right]^{2}+\gamma(1-\gamma) n_{e}\left[m_{1} m(L)\right]^{2}+ \\
& +d_{1} n_{e} \gamma m^{2}(L)+d(L) n_{e} \gamma m_{1}
\end{aligned}
$$

Based on the definition of the noise factor (1) and the mean and variance of each stage, the noise factor for the three stages of amplification can be written as:

$$
F=\gamma^{-1}\left(1+v_{r 1}+v_{r 2} / m_{1},\right.
$$

where $v_{r 1}=d_{1} / m_{1}^{2}$ is the relative variance of the distribution at the output of the second stage, and $v_{r 2}=d(L) / m^{2}(L)$ is the relative variance of the stage of amplification of a single electron.

If the second and third stages combined and considered to be one stage then the formula for the noise factor can be written as:

$$
F=\gamma^{-1}(1+D / M),
$$

where $M$ and $D$ are the mean gain and variance of the distribution at the output of the channel [5], [7].

Fig. 9 compares theoretical and experimental results of the noise factor as a function of the energy of the input electron beam. The computational results are obtained for the array of identical channels, and the ideal uniform emitter.

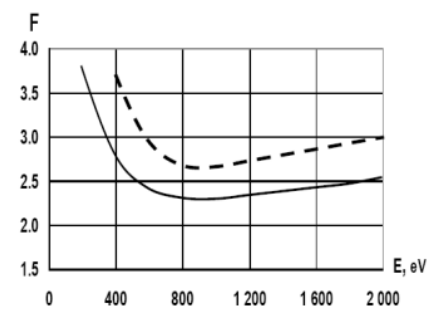

Fig. 9. Dependence of the noise factor $(F)$ on the energy (E) of the input electron beam (the solid curve represents calculations and dashed curve represents experiment).

\section{The Noise Factor of the Imaging Device}

The entire amplification process in the image converter and intensifier can be represented in the form of a sequence of several stages, where MCP can be considered as an intermediate stage with mean and variance $M_{2}$ and $D_{2}$ respectively.

Using the theorems about serial and parallel amplification stages, expressions for calculating the noise factor of the image detector can be obtained.

If $M_{1}, D_{1}$ and $M_{3}, D_{3}$ are the mean and variance of stages before (photocathode and/or EOS) and after (screen) MCP, then the noise factor of the whole device can be obtained, using the theorems about serial and parallel amplification stages:

$$
F=1+D_{1} \frac{1}{M_{1}^{2}}+D_{2} \frac{1}{M_{1} M_{2}^{2}}+D_{3} \frac{1}{M_{1} M_{2} M_{3}^{2}}
$$

This expression can be rearranged to the next formula:

$$
F=F_{1}+\frac{1}{M_{1}}\left(F_{2}-1\right)+\frac{1}{M_{1} M_{2}}\left(F_{3}-1\right),
$$

where $F_{1}, F_{2}$ and $F_{3}$ are the noise factors of previous, intermediate, and following stages. The last expression shows that to reduce the total noise, it is important not only reduce the noise of each stage, but also increase the mean gain.

\section{CONCLUSION}

Investigations dealing with reducing the noise factor and increasing the MTF of the image converters and intensifiers are of considerable practical interest, as they help to improve the visual acuity and range of vision of imaging devices.

The method and algorithm of evaluating the MTF of the EOS and MCP, proposed here, provide the opportunity to investigate the effect of parameters of the device on the MTF with the purpose to improve it.

The method of simulation of stochastic processes in the channel amplifier makes it possible to evaluate the noise factor of the MCP and the device, as a function of different parameters.

The theorems, proposed by the author, allows showing how the noise is transforming through the device, and how different multiplication stages contribute to the noise factor of the system.

The computational results show the correctness of the methods proposed by the author.

\section{ACKNOWLEDGMENT}

The author thanks V.N. Evdokimov for help in this work.

\section{REFERENCES}

[1] P. Schagen, "Image converters and intensifiers," J. Phys. E.: Sci.Instr, vol. 8, no. 3, pp. 153-160, 1975.

[2] V. Ivanov. (2009). The image quality evaluation. Micro-channel amplifiers, The University of Chicago, Argonne and Fermilab, Large-area picosecond photo-detectors project. [Online]. Available: http://psec.uchicago.edu/Papers/Ivanov\_image\_quality.doc

[3] P. Shagen, Advances in Image Pick-Up and Display, New York: Academic Press, 1974.

[4] A. V. Shymanska, "Computational modeling of stochastic processes in electron amplifiers,” J. Comput. Electron, vol. 9, pp. 93-102, June 2010.

[5] A. J. Guest, "A computer model of channel multiplier plate performance," Acta Electron, vol. 14, pp. 79-97, 1971.

[6] J. L. Wiza, "Microchannel plate detectors," Nucl. Instr. Methods, vol. 162 , pp. 587-601, 1979.

[7] A. V. Shymanska, "Numerical analysis of electron optical system with microchannel plate," J. Comput. Electron, vol. 10, no. 3, pp. 291-299, 2011.

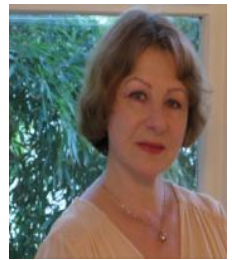

Alla Shymanska has BEng in electronics and $\mathrm{PhD}$ in mathematics and physics (St Petersburg). She has a long research and teaching experience in areas of physical electronics and charged particle optics at different research centers and universities in Russia and Ukraine, where she finished her career as an associate professor.

Currently Dr. Shymanska is a senior lecturer in the School of Computer and Mathematical Sciences of Auckland University of Technology (Auckland, New Zealand), where she teaches range of papers in mathematics and computer science. 